\title{
Short-term SSRI treatment normalises amygdala hyperactivity in depressed patients
}

\author{
B. R. Godlewska ${ }^{1}$, R. Norbury ${ }^{2}$, S. Selvaraj ${ }^{1}$, P. J. Cowen ${ }^{1}$ and C. J. Harmer ${ }^{1 *}$ \\ ${ }^{1}$ University Department of Psychiatry, Warneford Hospital, Oxford, UK \\ ${ }^{2}$ Oxford Centre for Clinical Magnetic Resonance Research (OCMR), John Radcliffe Hospital, Oxford, UK
}

\begin{abstract}
Background. Antidepressant drugs such as selective serotonin re-uptake inhibitors (SSRIs) remediate negative biases in emotional processing in depressed patients in both behavioural and neural outcome measures. However, it is not clear if these effects occur before, or as a consequence of, changes in clinical state.
\end{abstract}

Method. In the present study, we investigated the effects of short-term SSRI treatment in depressed patients on the neural response to fearful faces prior to clinical improvement in mood. Altogether, 42 unmedicated depressed patients received SSRI treatment (10 mg escitalopram daily) or placebo in a randomised, parallel-group design. The neural response to fearful and happy faces was measured on day 7 of treatment using functional magnetic resonance imaging. A group of healthy controls was imaged in the same way.

Results. Amygdala responses to fearful facial expressions were significantly greater in depressed patients compared to healthy controls. However, this response was normalised in patients receiving 7 days treatment with escitalopram. There was no significant difference in clinical depression ratings at 7 days between the escitalopram and placebotreated patients.

Conclusions. Our results suggest that short-term SSRI treatment in depressed patients remediates amygdala hyperactivity in response to negative emotional stimuli prior to clinical improvement in depressed mood. This supports the hypothesis that the clinical effects of antidepressant treatment may be mediated in part through early changes in emotional processing. Further studies will be needed to show if these early effects of antidepressant medication predict eventual clinical outcome.

Received 25 November 2011; Revised 6 March 2012; Accepted 8 March 2012; First published online 25 April 2012

Key words: amygdala, depression, emotional processing, fMRI, SSRIs.

\section{Introduction}

Patients with acute depression manifest a range of negative biases in the processing of emotional information, which are believed to contribute to the aetiology and maintenance of the depressed state (Beck, 2008). For example, compared to healthy controls, depressed patients selectively recall more negative, selfrelated emotional information in memory tasks (Bradley \& Matthews, 1983; Bradley et al. 1995) and demonstrate negatively biased perception of key social signals such as emotional facial expressions (Gur et al. 1992; Bouhuys et al. 1999). Such biases have been associated with aberrant responses across a network of neural areas involved in emotional processing, including an increased response of the amygdala to negative facial expressions in depressed patients

\footnotetext{
* Address for correspondence: Dr C. J. Harmer, University Department of Psychiatry, Warneford Hospital, Oxford OX3 7JX, UK.

(Email: catherine.harmer@psych.ox.ac.uk)
}

compared to matched controls (Sheline et al. 2001; Surguladze et al. 2004; Suslow et al. 2010; Victor et al. 2010).

We recently proposed that the therapeutic effect of antidepressant drugs could be mediated by early reversal of these negative emotional biases (Harmer et al. $2009 a$ ). For example, in healthy volunteers, 7 days treatment with the selective serotonin re-uptake inhibitor (SSRI), citalopram, and the selective noradrenaline re-uptake inhibitor, reboxetine, diminished the recognition of negative emotional faces, increased recall of positive self-referential words and attenuated the amygdala response to fearful faces as measured by functional magnetic resonance imaging (fMRI; Harmer et al. 2004, 2006; Norbury et al. 2007). Similarly, studies in depressed patients have shown attenuation of the amygdala response to sad and fearful facial expressions during SSRI treatment (Sheline et al. 2001; Fu et al. 2004; Victor et al. 2010). However, in these latter studies amygdala responses were measured at baseline and then again after 
8 weeks of SSRI treatment, by which time the therapeutic response had become established and subjective mood improved. Therefore, it is possible that remediation of negative biases in depressed patients during antidepressant treatment could be a consequence of resolution of depression rather than an early effect of drug treatment. In addition, the absence of a placebo control makes it difficult to exclude changes due to order effects during repeated scanning or non-specific effects of treatment.

Accordingly, the aim of the present study was to assess whether in depressed patients, SSRI treatment would remediate neural changes in emotional bias prior to the onset of clinical antidepressant effect. We used a single scan, between-subjects, placebocontrolled design to exclude order and non-specific effects. We predicted that, compared to placebo, shortterm SSRI treatment would attenuate amygdala responses to fearful faces in the absence of clinically important changes in mood ratings.

\section{Materials and method}

\section{Participants}

Altogether, 45 participants with major depression and 18 healthy controls completed the fMRI protocol. Controls were recruited through university volunteer lists and advertising in local newspapers. Depressed patients were recruited from a number of sources including newspaper advertisements $(n=35)$ as well as referral from other clinicians or researchers $(n=7)$. All participants were assessed for the presence of current and past psychiatric disorder with the Structured Clinical Interview for DSM-IV (Spitzer et al. 1995). The depressed patients met criteria for a primary diagnosis of major depressive disorder. Exclusion criteria for the study were the following. For depressed patients: suffering from psychosis or substance dependence as defined by DSM-IV; being at clinically significant risk of suicidal behaviour; having contraindications to escitalopram treatment or being treated with psychotropic medication $<3$ weeks before the study (5 weeks in the case of fluoxetine). For healthy volunteers: current or past history of Axis I disorder as defined by DSM-IV. For both groups: major somatic or neurological disorders; pregnancy or breast-feeding; contraindications to magnetic resonance imaging or concurrent medication, which could alter emotional processing. All participants were right-handed as assessed by the Edinburgh Handedness Inventory (Oldfield, 1971). The study was approved by Oxford Research Ethics Committee and all participants gave written informed consent. Participants were reimbursed for their time and any other expenses.

\section{Study design and drug treatment}

This was a double-blind, placebo-controlled, parallel group study where patients with depression were randomly assigned to receive $10 \mathrm{mg}$ escitalopram or placebo each morning for 7 days. Both groups were then scanned on the seventh day of treatment. Mood and anxiety were assessed using the Beck Depression Inventory (BDI; Beck et al. 1961), the Hamilton Depression Ratings Scale (HAMD; Hamilton, 1960) and the Spielberger's State-Trait Anxiety Inventory (Spielberger et al. 1983) immediately before starting treatment and on the seventh day of placebo/escitalopram. After the scan all patients were offered treatment openly with escitalopram according to usual clinical practice. The healthy controls were scanned without any pre-treatment. Their mood ratings were carried out just before the scan.

\section{Functional MRI data acquisition}

Functional MRI data were acquired in a total of 63 participants on a 3T Siemens TIM TRIO (Siemens AG, Erlangen, Germany), fitted with a body transmit coil and 12-channel head receive coil, located at the University of Oxford, Centre for Clinical Magnetic Resonance Research. Data were excluded from four participants due to technical difficulties $(n=2)$ and rapid head motion. The remaining 59 subjects [escitalopram-treated $(\mathrm{Esc})=21$, placebo-treated $(\mathrm{Pla})$ $=21$ and controls $(\mathrm{Con})=17]$ were included in the final analyses.

Functional MRI data were acquired with a voxel resolution of $3 \times 3 \times 3.5 \mathrm{~mm}, \mathrm{TR} / \mathrm{TE} / \mathrm{FA}=2000 \mathrm{~ms} /$ $28 \mathrm{~ms} / 87^{\circ}$. A total of 255 volumes were acquired in an experiment lasting $8.5 \mathrm{~min}$. Field maps were acquired using a dual echo 2D gradient echo sequence with echos at 5.19 and $7.65 \mathrm{~ms}$ and a repetition time of $444 \mathrm{~ms}$. Data were acquired on a $64 \times 64 \times 40$ grid, with a voxel resolution of $3 \mathrm{~mm}$ isotropic. $\mathrm{T}_{1}$-weighted structural images were acquired for subject alignment using a magnetisation prepared rapid acquisition by gradient echo sequence with the following parameters: voxel resolution $0.78 \times 0.8 \times 0.78 \mathrm{~mm}$ on a $208 \times 256 \times 200$ grid, TE $/ \mathrm{TI} / \mathrm{TR}=4.8 / 1100 / 2040 \mathrm{~ms}$.

\section{Functional MRI experimental task}

During fMRI scanning, participants completed a simple gender discrimination task involving the rapid presentation of fearful and happy facial expressions. In this task, nine 30-s blocks of a baseline fixation cross (condition A) were interleaved with eight 30-s blocks of the emotional task [four blocks of fear (condition B) and four blocks of happy (condition C)]. During each emotional block, participants viewed 10 emotional 
faces (five female) all derived from a standard set of pictures of facial affect (Matsumoto \& Ekman, 1988). Each face was presented for $100 \mathrm{~ms}$ and subjects were asked to report the gender of the face via a MRI compatible key pad. To ensure participants remained focused during the task within block interstimulus intervals (ISI) ranged between 2500 and $2900 \mathrm{~ms}$ (mean ISI $=2900 \mathrm{~ms}$ ). To reduce potential carry-over effects, cycles of alternation between conditions were counterbalanced across subjects. Thus, during the course of the 8.5-min experiment, half of the subjects completed the following order: ABACABACABACABACA, the remaining subjects ACABACABACABACABA. Stimuli were presented on a personal computer using E-Prime (version 1.0; Psychology Software Tools Inc., USA) and a cloned projection displayed to participants on an opaque screen located at the head of the scanner bore, which subjects viewed using angled mirrors. Subject responses were made via an MRI-compatible keypad. Stimulus presentation/subject button presses were registered and time-locked to fMRI data using E-Prime. Both accuracy (correct gender discrimination) and reaction times were recorded. Immediately before scanning, all subjects received training with another set of stimuli to ensure they fully understood the requirements of the task. Behavioural data were analysed using a repeated measures analysis of variance (ANOVA) model with group as the between-subjects factor and valence as the within-subjects factor implemented in SPSS v. 15 (SPSS Inc., USA).

\section{Functional MRI pre-processing and statistical analysis}

Functional MRI data were pre-processed and analysed using FSL, version 4.1.4 (Smith et al. 2004) Briefly, motion correction was applied using a rigid body registration to the central volume (Jenkinson et al. 2002). Brain matter was segmented from non-brain using a mesh deformation approach (Smith, 2002); Gaussian spatial smoothing was applied with a fullwidth half-maximum of $5 \mathrm{~mm}$; high pass temporal filtering was applied using a Gaussian-weighted running lines filter, with a $3 \mathrm{~dB}$ cut-off of $120 \mathrm{~s}$; susceptibility-related distortions were corrected as far as possible using field-map correction routines (Jenkinson et al. 2002).

A general linear model was fitted in pre-whitened data space (to account for autocorrelation in the fMRI residuals; Woolrich et al. 2001). Two explanatory variables (plus their temporal derivatives) were modelled: 'fear faces' and 'happy faces'. All explanatory variables were convolved with a default haemodynamic response function ( $\gamma$ function, delay $=6 \mathrm{~s}$, S.D. $=3 \mathrm{~s}$ ), and filtered by the same high pass filter as the data. Finally, each subject's estimated motion parameters (derived from the motion correction preprocessing step described above) were also included in the model as regressors of no interest. The full model was simultaneously regressed to the data, giving the best-fitting amplitudes for each explanatory variable. Individual subject data were then combined at the group level using a full mixed-effects analysis (Woolrich et al. 2004), including gender as covariate of no interest. This mixed-effects approach enables generalisation of the results beyond the sample of subjects tested. At the group level, individual participant 's first-level difference maps were compared within an amygdala region of interest [clusters determined by $\mathrm{Z}$ $>2.3$ and a (corrected) cluster significance threshold of $p=0.05]$, derived from the Harvard-Oxford anatomical atlas. When a statistically significant interaction was observed in the overall $3 \times 2$ analysis, this was decomposed by comparing each group to one another for the contrast fearful versus happy facial expressions within the amygdala region of interest. If these group (2) $\times$ emotion (2) interactions were significant, mean percentage signal change was extracted from the significant functional clusters and compared across groups using independent samples $t$ tests (performed using SPSS version 16.0). This small volume correction was also used to identify amygdala responses to face presentations in the healthy controls to compare effects of fearful versus happy facial expressions (main effect of emotion condition, see Supplementary material, S1).

Outside our amygdala a priori areas of interest, significant activations were identified using cluster-based thresholding of statistical images with a height threshold of $\mathrm{Z}>2.3$ and a (whole-brain corrected) spatial extent threshold of $p=0.05$. The results from this analysis are presented in the Supplementary material (S2).

\section{Pulsed arterial spin labelling}

Pulsed arterial spin labelling (PASL) data were also acquired on a subset of participants $(E s c=20, \mathrm{Pla}=16$ and Con =16). Using a flow-sensitive alternating inversion recovery labelling scheme, whole-brain volumes were acquired with a 3D gradient and spin echo imaging readout (MacIntosh et al. 2010). The imaging volume was $200 \mathrm{~mm} \times 200 \mathrm{~mm} \times 120 \mathrm{~mm}$ (half $\mathrm{kz}$ space coverage; $64 \times 64 \times 24$ matrix size; voxel dimensions: $3.1 \mathrm{~mm} \times 3.1 \mathrm{~mm} \times 5.0 \mathrm{~mm}$ ). The pulse repetition time and echo time were $3150 \mathrm{~ms}$ and $40.6 \mathrm{~ms}$, respectively. Background suppression of static tissue was achieved using two non-selective inversion pulses, prescribed at times that will null the signal from background grey and white matter and that have been 
shown to improve the temporal stability of the arterial spin labelling measurement. Optimal inversion null times were calculated for signals with $\mathrm{T}_{1}$ values of 700 and $1400 \mathrm{~ms}$, which is approximately consistent for white and grey matter, respectively, at 3T (Macintosh et al. 2010). A $5 \mathrm{~mm}$ full-width half-maximum spatial smoothing kernel filter was applied to the PASL images prior to sinc-interpolated subtraction between tag and control volumes. The top and bottom two slices in each PASL imaging volume were discarded due to wraparound in the $\mathrm{z}$ direction. The resulting perfusion maps were smoothed and demeaned prior to inclusion in the between-groups analysis (described above) as voxelwise covariates to minimise the potential impact of these variables on group comparisons.

We also performed a voxelwise between-group whole-brain analysis on resting perfusion images through repeated permutation (5000) of the observed data. Significant activations across the whole brain were identified using cluster-based thresholding of statistical images with a height threshold of $Z=2.3$ and a (whole-brain corrected) spatial extent threshold of $p<0.05$. Finally, amygdala regions of interest (ROIs) were registered to perfusion space to extract mean resting perfusion values $(\mathrm{ml} / 100 \mathrm{~g} / \mathrm{min})$ for comparison between-groups.

\section{Results}

\section{Demographic data and clinical ratings}

The patients randomized to receive escitalopram or placebo were well matched for age and gender. The Con group had a higher proportion of female participants, but not significantly so $\left(\chi^{2}=2.5, p=0.3\right.$; Table 1$)$. HAMD scores indicated that the patients were moderately depressed at baseline and a similar decline in scores was seen in both Esc and Pla groups over the 7 days of treatment (Table 1). Mean ( \pm S.E.M.) scores in the Esc group fell by $4.4 \pm 0.8$ v. $3.2 \pm 1.0$ in the placebo group $(t=0.86, p=0.4)$. Similarly, there were no significant difference in decline in scores on the BDI $(t=0.64, p=0.5)$ or state anxiety ratings $(t=-0.34$, $p=0.74$ ) between the two groups.

\section{Functional MRI behavioural performance}

Due to technical difficulties response accuracy and latency were not acquired for three patients $(n=2$, Esc). Subsequent analysis, therefore, included 19 Esc, 20 Pla and 17 Con participants. Subjects were highly accurate in their behavioural performance ( $>77.5 \%$ accuracy). Repeated measures ANOVA revealed no main effect of group or emotion and no group $\times$ emotion interaction for either response accuracy or latency (all $F^{\prime} s<1$, see Table 1).

\section{Functional MRI results}

A two-way repeated-measures ANOVA model in FSL comparing haemodynamic differences across groups [Esc, Pla and Con and conditions (fear and happy) revealed a significant group $\times$ condition interaction in right amygdala (Fig. 1)]. To decompose this significant interaction, we compared, separately, Pla depressed patients and controls (Fig. 2a), drug treated patients with Pla patients (Fig. $2 b$ ) and drug-treated patients with controls within this amygdala region using FSL. These simple main effect analyses revealed greater BOLD response to fear in Pla vs. Esc $\left(t_{40}=2.729\right.$, $p=0.009$; Fig. $2 a)$ and Pla vs. Con $\left(t_{28.39}=3.51\right.$, $p=0.001$; Fig. $2 b$ ). By contrast, the BOLD response to happy faces was similar across groups (all $p^{\prime}$ s $>0.5$ ). Between-group differences in right amygdala BOLD response were not affected by adding resting perfusion maps as a voxelwise covariate. Similarly, no significant differences were observed in resting brain perfusion either at the whole-brain level or using a ROI-based analysis of the right amygdala (all $p^{\prime}$ s $>0.2)$.

Consistent with the prediction that these changes in neural response do not relate to immediate changes in mood or anxiety with antidepressant treatment, there were no significant associations between change in HAMD, BDI and State Anxiety with the response in the amygdala to fear in the Esc group (all $p$ values $>0.24)$.

\section{Discussion}

The principal finding of our study is that amygdala response to fearful faces was significantly lower in depressed patients treated with 7 days escitalopram than in those receiving placebo. Ratings of depression or anxiety did not distinguish placebo and drug-treated groups at this point, suggesting that the attenuation in amygdala responsivity was caused by SSRI administration rather than change in clinical state. Consistent with this, there was no association between the change in ratings during treatment and the magnitude of the amygdala response to fearful facial expressions. Further, this effect of SSRI treatment cannot be explained by differences in basal perfusion of the amygdala measured with arterial spin labelling. Finally, the between-subjects placebo controlled design indicates that the differences in amygdala reactivity during SSRI treatment were not due to an effect of repeated fMRI examination or because of non-specific placebo effects.

These findings in SSRI-treated depressed patients are similar to those we noted in a similarly designed, placebo-controlled study of healthy volunteers, where 
Table 1. Demographic and clinical characteristics of participants

\begin{tabular}{|c|c|c|c|}
\hline $\begin{array}{l}\text { Variable } \\
(\text { mean } \pm \text { s.D. })\end{array}$ & $\begin{array}{l}\text { Placebo-treated } \\
(n=21)\end{array}$ & $\begin{array}{l}\text { Escitalopram-treated } \\
(n=21)\end{array}$ & $\begin{array}{l}\text { Healthy controls } \\
(n=17)\end{array}$ \\
\hline Age (years) & $31.1 \pm 12.1$ & $32.0 \pm 10.7$ & $33.7 \pm 12.3$ \\
\hline Male/Female & $10 / 11$ & $9 / 12$ & $4 / 13$ \\
\hline Male/Female age & $\begin{array}{c}31.4 \pm 10.4 / \\
30.8 \pm 4.2\end{array}$ & $\begin{array}{r}33.5 \pm 11.3 / \\
30.8 \pm 10.6\end{array}$ & $\begin{array}{r}34.4 \pm 17.4 / \\
33.5 \pm 11.2\end{array}$ \\
\hline HAMD before treatment (mean \pm S.D.) & $23.3 \pm 4.5$ & $24.2 \pm 5.7$ & $0.5 \pm 0.7$ \\
\hline HAMD after treatment (mean \pm S.D.) & $20.0 \pm 4.3$ & $19.8 \pm 7.8$ & \\
\hline BDI before treatment (mean \pm s.D.) & $30.9 \pm 9.6$ & $30.9 \pm 9.4$ & $1.1 \pm 1.3$ \\
\hline BDI after treatment (mean \pm S.D.) & $23.8 \pm 9.51$ & $25.4 \pm 11.8$ & \\
\hline STAI-S before treatment (mean \pm S.D.) & $52.4 \pm 12.45$ & $55.6 \pm 11.7$ & $27.5 \pm 5.2$ \\
\hline STAI-S after treatment (mean \pm S.D.) & $46.7 \pm 10.23$ & $50.7 \pm 11.2$ & \\
\hline Mean age at onset of depression (range) & $24(12-45)$ & $23.5(12-47)$ & \\
\hline Mean number of episodes (range) & $2.5(1-10)$ & $3(1-10)$ & \\
\hline $\begin{array}{l}\text { Mean months duration of current } \\
\text { episode (range) }\end{array}$ & $9.5(1-36)$ & $13.4(1-36)$ & \\
\hline Medication naive $(n)$ & 11 & 10 & \\
\hline $\begin{array}{l}\text { Co-morbidities (number of } \\
\text { patients, condition) }\end{array}$ & $\begin{array}{l}1 \text { - somatisation } \\
\text { disorder }\end{array}$ & $\begin{array}{l}1 \text { - trichillomania } \\
2 \text { - GAD } \\
1 \text { - social anxiety } \\
\text { disorder }\end{array}$ & \\
\hline $\begin{array}{l}\text { Gender discrimination } \\
\text { accuracy } \% \text { (fear) }\end{array}$ & $96.58 \pm 5.54$ & $96.37 \pm 3.84$ & $97.35 \pm 3.24$ \\
\hline $\mathrm{RT}$, ms (fear) & $756.48 \pm 130.91$ & $725.45 \pm 165.81$ & $680.44 \pm 146.48$ \\
\hline $\begin{array}{l}\text { Gender discrimination } \\
\text { accuracy \% (happy) }\end{array}$ & $97.92 \pm 3.35$ & $96.62 \pm 3.74$ & $98.38 \pm 1.75$ \\
\hline RT, ms (happy) & $760.38 \pm 141.17$ & $720.96 \pm 170.92$ & $680.99 \pm 164.30$ \\
\hline
\end{tabular}

HAMD, Hamilton Rating Scale for Depression; BDI, Beck Depression Inventory; STAI-S, State-Trait Anxiety Inventory State; $\mathrm{GAD}$, generalised anxiety disorder; RT, reaction time.

7 days treatment with the SSRI, citalopram, diminished amygdala responses to fearful facial expressions (Harmer et al. 2006). The latter study used backward masking of emotional faces, which were presented below the threshold of conscious awareness. While the emotional stimulus presentations used in the present investigation were not masked, the nature of the task could still be considered 'implicit' and therefore likely to activate limbic regions implicated in rapid automatic appraisal of emotional stimuli (Killgore \& Yurgelen-Todd, 2004).

Our findings are also consistent with other studies of antidepressant treatment in depressed patients, which have used fMRI to examine neural responses to fearful and sad faces during more extended periods of SSRI administration. For example, Sheline et al. (2001) found that the amygdala response to masked fearful faces was reduced bilaterally relative to baseline in patients treated with sertraline in doses of approximately $100 \mathrm{mg}$ daily for 8 weeks. Victor et al. (2010), employing a similar treatment regimen, found that sertraline treatment lowered responses to masked sad faces in the right amygdala and Fu et al. (2004) found that 8 weeks fluoxetine treatment ( $20 \mathrm{mg}$ daily) decreased responses in left amygdala to implicitly presented sad faces of increasing emotional intensity. In these studies, however, most patients had shown clinically significant symptomatic improvement by the time of the second fMRI scan. The neurochemical mechanisms by which SSRIs might lower amygdala responses to fear are not presently clear. The amygdala receives a substantial serotonergic input from the dorsal raphe and, in animals, application of serotonin to amygdala interneurones increases release of $\gamma$-aminobutyuric acid, which, in turn, modifies the activity of glutamatergic projection neurones (Rainnie, 1999). Thus, changes in serotonin neurotransmission are well placed to alter the excitability of internal amygdala networks. However, the serotonin receptor subtypes involved and their location with regard to different neuronal types are not fully elucidated.

Taken in conjunction with the above studies, our findings indicate that the attenuating effects of SSRIs on amygdala responses to negative facial expressions in depressed patients can occur early in treatment, prior to meaningful symptomatic improvement. 


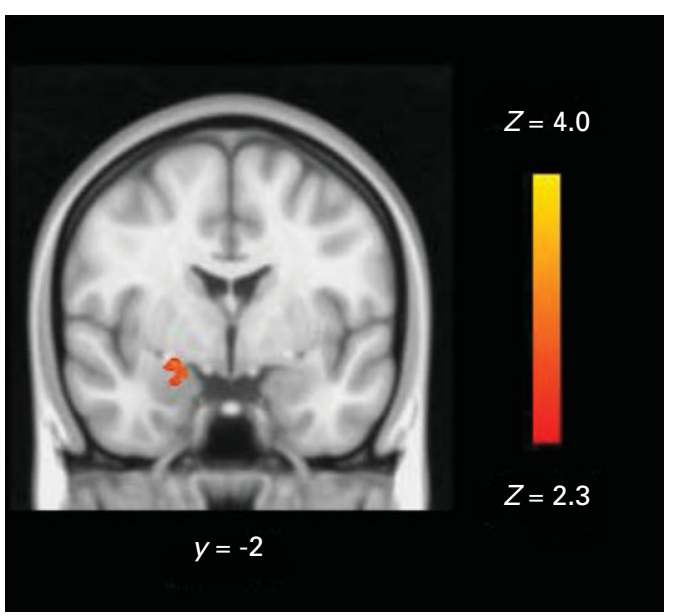

Fig. 1. Coronal image depicting the group $\times$ emotion interaction in right amygdala. The image is thresholded at $Z=2.3, p<0.05$ (small volume correction). Lower numeral refers to the coordinate in Montreal Neurological Institute space.

(a)

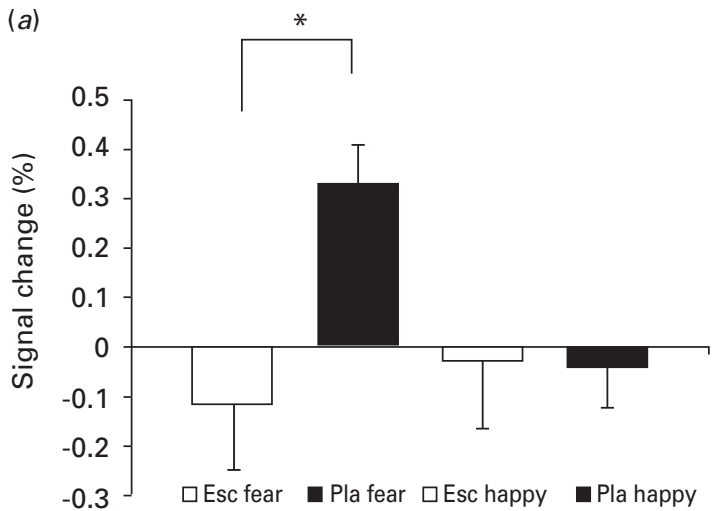

(b)

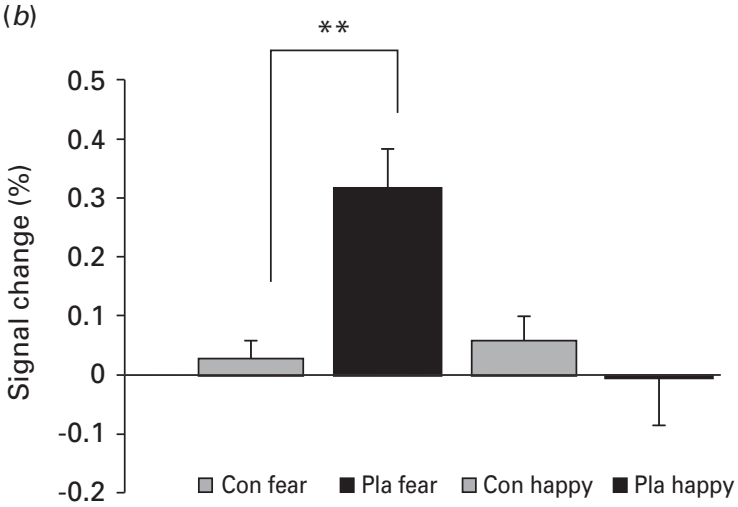

Fig. 2. Plot depicting BOLD activation (expressed as \% signal change) to fearful and happy facial expressions in right amygdala for (a) escitalopram- (Esc) and placebo-treated (Pla) participants, ${ }^{*} p=0.009$ and (b) for healthy controls (Con) and Pla participants, ${ }^{* *} p=0.001$. Bars show mean, error bars standard error.
Indeed, in healthy subjects we found that both citalopram (Murphy et al. 2009) and the atypical antidepressant, mirtazapine (Rawlings et al. 2010), reduced amygdala responses to fear a few hours after administration of single therapeutic dose. The translation of these findings from healthy volunteers to an acutely ill patient group suggests that neuropsychologically relevant, remediating effects of antidepressant treatment on emotional processing may be present very shortly after initiation of treatment in depressed patients. Furthermore, in a behavioural study, we have recently found that a single dose of reboxetine in depressed patients reversed negative emotional biases in both facial expression recognition and emotional memory in the absence of any change in subjective mood (Harmer et al. 2009b).

In the present study, the effect of escitalopram to lower fear responses relative to placebo was apparent in the right amygdala rather than bilaterally and in our antidepressant studies in healthy volunteers, using the same or similar paradigms, changes in fear processing have been most apparent in the right amygdala (Harmer et al. 2006; Murphy et al. 2009; Rawlings et al. 2010). However, the investigations of antidepressants in depressed patients outlined above do not apparently reveal a consistent pattern in this respect and it is possible that the detection of laterality is influenced by a number of additional factors, such as the nature of the task and the number of trials presented. We also saw here a specific effect of SSRI treatment on response to negative facial expressions, which is the best replicated effect seen early and late in treatment (see Harmer et al. 2011). However, some studies have also revealed increased amygdala responses to happy facial expressions after antidepressant treatment (Norbury et al. 2009; Rawlings et al. 2010; Victor et al. 2010), which were not detected here. The reason for this discrepancy is unclear but could involve differences between drug treatments, patient or volunteer characteristics and explicit versus implicit emotional paradigms. Nonetheless, these findings are all in line with a decrease in the relative processing of negative versus positive stimuli at the level of the amygdala.

We also studied a group of age-matched healthy controls with the same facial expression task, but it must be noted that control participants did not receive drug or placebo treatment prior to scanning. In addition, there were proportionately more women than men in the Con group than in the patient groups, leading us to enter gender as a covariate in the fMRI analysis (see Method). These differences limit the conclusions that can be drawn from the comparison between the Pla depressed group and healthy volunteer group, although our findings are consistent with a number of other imaging studies that have found 
increased amygdala responses to negative facial expressions in depressed patients relative to healthy controls (Sheline et al. 2001; Surguladze et al. 2004; Suslow et al. 2010; Victor et al. 2010). A major aim of our study was to exclude an effect of repeated scanning and placebo effects in changes in amygdala response in drug-treated patients and we therefore used a single scan, between-subjects placebo-controlled design. However, this approach is open to the objection that the two patient groups may have differed in baseline amygdala reactivity to fearful faces. In as far as we could ascertain, the patients randomised to placebo and escitalopram were similar in terms of personality, clinical and demographic factors, which could have altered the response. However, future studies using a within-subjects design are warranted to explore this question further and to explore whether this early amygdala response is predictive, as hypothesised, of later clinical response.

BOLD magnitude is determined by the interaction between increases in cerebral blood flow, cerebral blood volume and rate of oxygen consumption (Brown et al. 2003; Buxton et al. 2004) and is affected by differences in resting cerebral blood flow (Brown et al. 2003). Characterising the effects of escitalopram (or any drug) upon task-specific brain activity may, therefore, be confounded by drug-related effects on baseline activity. Arguing against this interpretation of our data, we found no significant between-groups differences in resting perfusion using either wholebrain or ROI-based approaches, indicating a degree of region and task specificity unlikely to be related to global effects of escitalopram on baseline blood flow.

An unexpected finding was that the current sample of healthy volunteers did not show increased amygdala response to fearful versus happy facial expressions in the statistically significant cluster of interest. However, examination of this within-subjects difference may not be justified in studies where the data derived from the amygdala have been identified through the statistical comparison of depressed and healthy controls. In these circumstances, a difference between response to emotions in the healthy volunteer group may be an artefact of the comparison used to identify the difference between the groups rather than a pattern that will be seen consistently across the whole, or other parts of, the amygdala. Consistent with this, analysis of the amygdala region of interest in healthy volunteers alone revealed a trend for the pattern expected by results in meta-analyses of amygdala responsivity in healthy people [increased responses to fearful compared to happy facial expressions at $p=0.1$, see Supplementary material S1 (e.g. Phan et al. 2002)].
The apparent rapid resolution of negative emotional processing biases by antidepressant administration in depressed patients supports the notion that such an effect precedes the overt clinical effects of antidepressant treatment and may be a relevant therapeutic mechanism. According to this view, remediation of negative emotional bias is translated into improved subjective mood over time as individuals re-experience and relearn personal and social contingencies in an implicitly transformed emotional world (Harmer et al. 2009a). If this hypothesis is correct, one might expect that the early changes in emotional bias produced by antidepressant treatment would predict eventual therapeutic outcome. Although, as yet, there are no data exploring this question with functional neuroimaging outcomes measures, behavioural changes in emotional processing with antidepressant drug treatment have been associated with clinical response 4 weeks later (Tranter et al. 2009). It is also of great interest that certain negative biases in emotional processing may persist in recovered unmedicated patients who demonstrate abnormalities in behavioural and neural responses similar to those apparent in acute depression (Bhagwagar et al. 2004; Victor et al. 2010). It would be predicted that such biases should be attenuated by continued antidepressant treatment (Sheline et al. 2001; Fu et al. 2004; Victor et al. 2010). This may provide a mechanism for the established efficacy of antidepressant treatment in longer-term maintenance therapy and a possible means of identifying patients who require it.

\section{Supplementary material}

For supplementary material accompanying this paper, visit http:/ /dx.doi.org/10.1017/S0033291712000591.

\section{Acknowledgement}

The study was sponsored by the UK Medical Research Council (Grant number G0701421).

\section{Declaration of Interest}

C.J.H. is on the advisory board of P1vital and has received consultancy fees from Servier, P1vital, Lundbeck, and Merck-Sharpe and Dohme. She holds shares in P1vital Ltd and is a company director of Oxford Psychologists Ltd. P.J.C. has been a paid member of advisory boards of Eli Lilly, Lundbeck, Servier and Wyeth. He has received remuneration for scientific advice given to legal representatives of GlaxoSmithKline. 


\section{References}

Beck AT (2008). The evolution of the cognitive model of depression and its neurobiological correlates. American Journal of Psychiatry 165, 969-977.

Beck AT, Ward CH, Mendelson M, Mock J, Erbaugh J (1961). An inventory for measuring depression. Archives of General Psychiatry 4, 561-571.

Bhagwagar Z, Cowen PJ, Goodwin GM, Harmer CJ (2004). Normalization of enhanced fear recognition by acute SSRI treatment in subjects with a previous history of depression. American Journal of Psychiatry 161, 166-168.

Bouhuys AL, Geerts E, Gordijn MC (1999). Depressed patients perceptions of facial emotions in depressed and remitted states are associated with relapse: a longitudinal study. Journal of Nervous Mental Disorder 187, 595-602.

Bradley B, Matthews A (1983). Negative self-schemata in clinical depression. Clinical Psychology 22, 173-181.

Bradley BP, Mogg K, Williams R (1995). Implicit and explicit memory for emotion-congruent information in clinical depression and anxiety. Behavioural Research and Therapeutics 33, 755-770.

Brown GG, Eyler Zorrilla LT, Georgy B, Kindermann SS, Wong EC, Buxton, RB (2003). BOLD and perfusion response to finger-thumb apposition after acetazolamide administration, differential relationship to global perfusion. Journal of Cerebral Blood Flow and Metaboloism 23, 829-837.

Buxton RB, Uludag K, Dubowitz DJ, Liu TT (2004). Modeling the hemodynamic response to brain activation. Neuroimage 23, S220-S233.

Fu CH, Williams SC, Cleare AJ, Brammer MJ, Walsh ND, Kim J, Andrew CM, Pich EM, Williams PM, Reed LJ, Mitterschiffthaler MT, Suckling J, Bullmore ET (2004). Attenuation of the neural response to sad faces in major depression by antidepressant treatment. A prospective, event related functional magnetic resonance imaging study. Archives of General Psychiatry 61, 877-889.

Gur RC, Erwin RJ, Gur RE, Zwil AS, Heimberg C, Kraemer HC (1992). Facial emotion discrimination, II, behavioural findings in depression. Psychiatry Research 42, 241-251.

Hamilton M (1960). A rating scale for depression. Journal of Neurology and Neurosurgery 23, 56-62.

Harmer CJ, Cowen PJ, Goodwin GM (2011). Efficacy markers in depression. Journal of Psychopharmacology 25, 1148-1158.

Harmer CJ, Goodwin GM, Cowen PJ (2009a). Why do antidepressants take so long to work? A cognitive neuropsychological model of antidepressant drug action. British Journal of Psychiatry 195, 102-108.

Harmer CJ, Mackay CE, Reid CB, Cowen PJ, Goodwin GM (2006). Antidepressant drug treatment modifies the neural processing of nonconcious threat cues. Biological Psychiatry 59, 816-820.

Harmer CJ, O'Sullivan U, Favaron E, Massey-Chase R, Ayres R, Reinecke A, Goodwin GM, Cowen PJ (2009b). Effect of acute antidepressant administration on negative affective bias in depressed patients. American Journal of Psychiatry 166, 1178-1184.
Harmer CJ, Shelley NC, Cowen PJ, Goodwin GM (2004). Increased positive versus affective perception and memory in healthy volunteers following selective serotonin and norepinephrine reuptake inhibition. American Journal of Psychiatry 161, 1256-1263.

Jenkinson M, Bannister P, Brady M, Smith S (2002). Improved optimization for the robust and accurate linear registration and motion correction of brain images. Neuroimage 12, 825-841.

Killgore WD, Yurgelen-Todd DA (2004). Activation of the amygdala and anterior cingulate during nonconscious processing of sad versus happy faces. Neuroimage 21, 1215-1223.

MacIntosh BJ, Filippini N, Chappell MA, Woolrich MW, Mackay CE, Jezzard P (2010). Assessment of arterial arrival times derived from multiple inversion time pulsed arterial spin labelling MRI. Magnetic Resonance in Medicine 63, 641-647.

Matsumoto J, Ekman P (1988). Japanese and Caucasian Facial Expressions of Emotion (JACFEE) and Neutral Faces (JACNeuf). Human Interaction Laboratory: San Francisco.

Murphy S, O'Sullivan U, Cowen PJ, Harmer CJ (2009). Effect of a single dose of citalopram on amygdala response to emotional faces. British Journal of Psychiatry 194, 535-540.

Norbury R, Taylor MJ, Selvaraj S, Murphy SE, Harmer CJ, Cowen PJ (2009). Short-term antidepressant treatment modulates amygdala response to happy faces. Psychopharmacology 206, 197-204.

Norbury R, Mackay CE, Cowen PJ, Goodwin GM, Harmer CJ (2007). Short-term antidepressant treatment and facial processing, Functional magnetic resonance imaging study. British Journal of Psychiatry 90, 531-532.

Oldfield RC (1971). The assessment and analysis of handedness, the Edinburgh inventory. Neuropsychologia. 9, 97-113.

Phan KL, Wager T, Taylor SF, Liberzon I (2002). Functional neuroanatomy of emotion, a meta-analysis of emotion activation studies in PET and fMRI. Neuroimage 16, 331-348.

Rainnie DG (1999). Serotonergic modulation of neurotransmission in the rat basolateral amygdala. Journal of Neurophysiology 82, 69-85.

Rawlings NB, Norbury R, Cowen PJ, Harmer CJ (2010). A single dose of mirtazapine modulates neural responses to emotional faces in healthy people. Psychopharmacology 212, 625-634.

Sheline YI, Barch DM, Donnelly JM, Ollinger JM, Snyder, AZ, Mintun, MA (2001). Increased amygdala response to masked emotional faces in depressed subjects resolves with antidepressant treatment, An FMRI study. Biological Psychiatry 50, 651-658.

Smith S, Jenkinson M, Woolrich MW, Beckman CF, Behrens TEJ, Johansen-Berg H, Bannister PR, de Luca M, Drobnjak I, Flitney DE, Niazy RK, Saunders J, Vickers J, Zhang Y, de Stefano N, Brady JM, Matthews PM (2004). Advances in functional and structural MR image analysis and implementation as FSL. Neuroimage 23, S208-S219.

Smith SM (2002). Fast robust automated brain extraction. Human Brain Mapping 17, 143-155. 
Spielberger CD, Gorssuch RL, Lushene PR, Vagg PR, Jacobs GA (1983). Manual for the State-Trait Anxiety Inventory. Consulting Psychologists Press, Inc. : Mountain View, CA.

Spitzer RL, Williams JBW, Gibbon M, First MB (1995). Structured Clinical Interview for DSM-1V (SCID). New York State Psychiatric Institute Biometrics Research: New York.

Surguladze SA, Young AW, Senior C, Brebion G,

Travis MJ, Phillips ML (2004). Recognition accuracy and response bias to happy and sad facial expressions in patients with major depression. Neuropsychology 18, 212-218.

Suslow T, Konrad C, Kugel H, Rumstadt D, Zwitserlood P, Schöning S, Ohrmann, P, Bauer J, Pyka M, Kersting A, Arolt, V, Heindel W, Dannlowski U (2010). Automatic mood congruent amygdala responses to masked facial expressions in major depression. Biological Psychiatry 67, 155-160.
Tranter R, Bel D, Harmer CJ, Healey D, Anderson IM (2009). The effect of serotonergic and noradrenergic antidepressants on face emotion processing in depressed patients. Journal of Affective Disorders 118, 87-93.

Victor TA, Furey ML, Fromm SJ, Öhman A, Drevets, WC (2010). Relationship between amygdala responses to masked faces and mood state and treatment in major depressive disorder. Archives of General Psychiatry 67, 1128-1138.

Woolrich MW, Behrens TE, Beckmann CF, Jenkinson M, Smith SM (2004). Multilevel linear modelling for FMRI group analysis using Bayesian inference. Neuroimage 21, 1732-1747.

Woolrich MW, Ripley BD, Brady M, Smith SM (2001).

Temporal autocorrelation in univariate linear modelling of FMRI data. Neuroimage 14,

1370-1386. 